\title{
Label-Free Visualization of Early Cancer Hepatic Micrometastasis and Intraoperative Image-Guided Surgery by Photoacoustic Imaging
}

\author{
Qian $\mathrm{Yu}^{* 1}$, Shanshan Huang*1, Zhiyou $\mathrm{Wu}^{1}$, Jiadi Zheng ${ }^{2}$, Xiaoyuan $\mathrm{Chen}^{3}$, and Liming $\mathrm{Nie}^{1}$ \\ ${ }^{I}$ State Key Laboratory of Molecular Vaccinology and Molecular Diagnosis and Center for Molecular Imaging and Translational \\ Medicine, School of Public Health, Xiamen University, Xiamen, China; ${ }^{2}$ Department of Neurosurgery, Xiamen Hospital, Beijing \\ University of Chinese Medicine, Xiamen, China; and ${ }^{3}$ Laboratory of Molecular Imaging and Nanomedicine, National Institute of \\ Biomedical Imaging and Bioengineering, National Institutes of Health, Bethesda, Maryland
}

The detection of cancer micrometastasis for early diagnosis and treatment poses a great challenge for conventional imaging techniques. The aim of our study was to evaluate the performance of photoacoustic imaging (PAI) in detecting hepatic micrometastases from melanoma at a very early stage and in aiding tumor resection by intraoperative guidance. Methods: In vivo studies were performed by following protocols approved by the Ethical Committee for Animal Research at Xiamen University. First, a mouse model of B16 melanoma metastatic to the liver $(n=10)$ was established to study the development of micrometastases in vivo. Next, the mice were imaged by a scalable PAl instrument, ultrasound, 9.4-T highresolution $\mathrm{MRI}, \mathrm{PET} / \mathrm{CT}$, and bioluminescence imaging. PAI scans acquired with optical wavelengths of $680-850 \mathrm{~nm}$ were kept spectrally unmixed by using a linear least-squares method to differentiate various components. Differences in signal-to-background ratios among different modalities were determined with the 2-tailed paired $t$ test. The diagnostic results were assessed with histologic examination. Excised liver samples from patients diagnosed with hepatic cancer were also examined to identify the tumor boundaries. Surgical removal of metastatic melanoma was precisely guided in vivo by the portable PAl system. Results: PAI was able to detect metastases as small as approximately $400 \mu \mathrm{m}$ at a depth of up to $7 \mathrm{~mm}$ in vivo-a size that is smaller than can be detected with ultrasound and MRI. The tumor-to-liver ratio for PAl at $8 \mathrm{~d}(4.2 \pm 0.2, n=6)$ and $14 \mathrm{~d}(9.2 \pm 0.4, n=5)$ was significantly higher than for PET/CT (1.8 \pm $0.1, n=5$, and $4.5 \pm 0.2, n=5$, respectively; $P<0.001$ for both). Functional PAl revealed dynamic oxygen saturation changes during tumor growth. The limit of detection was approximately $219 \mathrm{cells} / \mu \mathrm{L}$ in vitro. We successfully performed intraoperative PAl-guided surgery in vivo using the portable PAI system. Conclusion: Our findings offer a rapid and effective complementary clinical imaging application to noninvasively detect micrometastases and guide intraoperative resection.

\footnotetext{
Received Jul. 15, 2019; revision accepted Nov. 11, 2019.

For correspondence or reprints contact: Liming Nie, State Key Laboratory of Molecular Vaccinology and Molecular Diagnosis and Center for Molecular Imaging and Translational Medicine, School of Public Health, Xiamen University, Xiang'an S. Rd., Xiang'an District, Xiamen 361102, P.R. China. E-mail: nielm@xmu.edu.cn.

${ }^{*}$ Contributed equally to this work

Published online Dec. 5, 2019.

COPYRIGHT (C 2020 by the Society of Nuclear Medicine and Molecular Imaging.
}

Key Words: micrometastasis; visualization; early stage; intraoperative navigation; photoacoustic imaging

J Nucl Med 2020; 61:1079-1085

DOI: 10.2967/jnumed.119.233155

$\mathbf{M}$ elanoma is a malignant tumor of the skin that has shown a steady increase in incidence over the past few decades (1). Melanoma metastasis consists of a consecutive series of steps, including exfoliation from primary tumors, survival of tumor cells in the circulation, staying in new organs, exudation to surrounding tissues, and angiogenesis of metastatic tumors, which ultimately proliferate in a new environment (2,3). Malignant melanoma cells shed from the primary tumor often metastasize to the lymph nodes (4), liver (5), lungs (6), and brain (7). The liver is the most frequently involved organ by metastatic choroidal melanoma (8). $\mathrm{Cu}-$ taneous melanoma metastasizes to the liver less frequently, but $15 \%-20 \%$ of metastatic melanoma still occurs in the liver (9). The survival rate for patients with melanoma metastatic to the liver has been reported to be low (10). Because of its aggressive progression and high metastasis risk, melanoma is associated with a poor prognosis, as well as high morbidity and mortality rates (11). Therefore, accurate diagnosis of micrometastasis to the liver at an early stage is critical to improve the prognosis and survival rate.

Metastasis is usually detected clinically by imaging methods such as ultrasound, MRI, and PET/CT. Ultrasound imaging lacks sufficient contrast in soft tissue and cannot detect blood oxygenation, and MRI is not the primary choice for the liver because of its suboptimal sensitivity, long scanning time, and high operational costs. PET is useful for detecting cancer metastasis and monitoring therapeutic response, but ${ }^{18} \mathrm{~F}-\mathrm{FDG}$ may yield false-positive results in patients with other symptoms and has a high examination fee (12). Therefore, the current clinical imaging modalities are not ideal for detecting small tumors and micrometastasis.

Photoacoustic imaging (PAI) is a noninvasive method that combines the high spatial resolution of ultrasound with the rich contrast of optical imaging (13). It allows high-precision imaging and characterization of molecules, cells, tissues, and organs (14) so that features can be extracted from PAI scans (15). PAI can provide endogenous contrast among different anatomic structures on account of intrinsic light absorbers such as hemoglobin (16), lipid (17), and 
melanin (18). Ex vivo PAI of melanin in the resected sentinel lymph nodes shows an excellent correlation with histologic melanoma cell infiltration (19). In addition, in vivo PAI can track unmarked circulating melanoma cells, can image functional connections between blood vessels and the whole brain (20), and can quantify the blood flow and oxygen metabolism of lesions (21). It combines the advantages of high resolution, high sensitivity, and deep penetration, enabling early diagnosis of hepatic micrometastasis.

The high recurrence rate after tumor resection is due to incomplete removal of the malignant mass, which results in residual cancer cells. Surgeons rely primarily on visual and tactile identification of cancerous tissue to distinguish it from benign tissue, a method that is often inaccurate. In the liver, PAI possesses optimal resolution and imaging depth that are far superior to the limited resolution and imaging depth of fluorescence imaging (22). As a label-free technique, PAI obviates the shortcomings of the widely used fluorescence imaging, which requires gene expression or injection of fluorescence probes $(23,24)$. PAI offers excellent spatial and temporal resolution to accurately determine tumor margins and tissue structures (25) and is therefore a viable option for early-stage cancer detection. In addition, its portable setup and handheld probe save time and labor.

Resection is the basic method of treatment for tumors that are metastatic to the liver, and it offers the optimal long-term survival rate. A previous study combined MRI, PAI, and Raman imaging to guide intraoperative tumor resection by intravenous injection of nanoparticles (26). Ideally, a label-free noninvasive portable device is needed in the operating room. In particular, the device needs to be designed for easy intraoperative navigation and real-time imaging. The combination of high-resolution, fast, and portable PAI would enable accurate diagnosis and resection for micrometastatic tumors. Further development of such a device would imply future improvements in PAI-guided tumor surgery and in patient prognosis (27).

Here, we conducted a comprehensive study to accurately detect tumor micrometastases in living animals and clinical tissue specimens without labeling by high-resolution PAI systems (Fig. 1). Our results showed that PAI technique is somewhat comparable to conventional

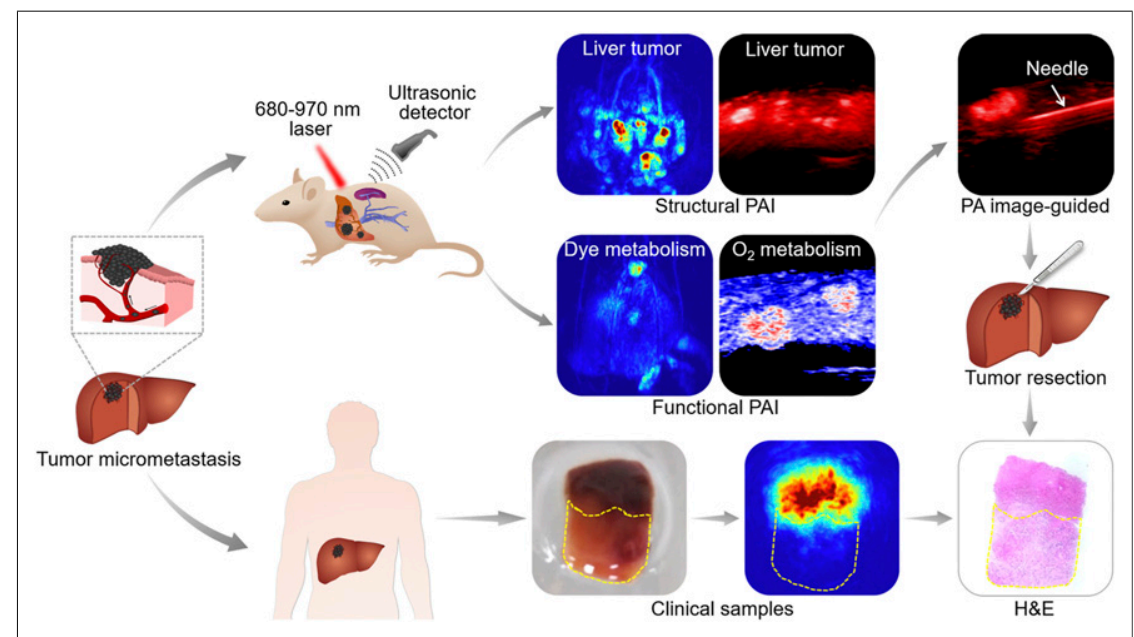

FIGURE 1. Workflow of PAI for melanoma hepatic-metastasis detection, profiling, and resection for animal models and clinical samples. Two kinds of hepatic metastasis models were established by injecting B16 tumor cells directly into mouse hepatic lobe and subcapsular spleen. B16 cells entered liver tissue through hepatic portal vein and developed into tumors. Tumor samples were used for $\mathrm{PAl}$ and were pathologically verified. $\mathrm{H} \& \mathrm{E}=$ hematoxylin and eosin staining; $\mathrm{PA}=$ photoacoustic. imaging technologies such as MRI and PET, permitting clinical application for early diagnosis of tumors and guiding intraoperative resection.

\section{MATERIALS AND METHODS}

The study timeline is shown in Supplemental Figure 1 (supplemental materials are available at http://jnm.snmjournals.org).

\section{Cell Lines and Culture}

Luciferase-expressing melanoma B16 and hepatoma HepG2 cell lines were maintained in Dulbecco modified Eagle medium (Sigma-Aldrich) supplemented with $10 \%$ fetal bovine serum (Sigma-Aldrich), a $100 \mathrm{U} / \mathrm{mL}$ concentration of penicillin, and a $100 \mathrm{mg} / \mathrm{mL}$ concentration of streptomycin, at $37^{\circ} \mathrm{C}$ with $5 \% \mathrm{CO}_{2}$. One hour before injection into mice, $90 \%-$ $100 \%$ confluent cultures were harvested by trypsinization $(0.25 \%$ trypsin and $0.02 \%$ ethylene diamine tetraacetic acid in sterile phosphate-buffered saline [PBS]) and resuspended in the sterile PBS to final densities.

\section{Establishment of Tumor Xenografts}

All animal experiments were performed in accordance with the relevant guidelines and regulations. The study protocols were approved by the Ethical Committee for Animal Research at Xiamen University. Six- to 8-wk-old male immunodeficient nude mice were purchased from the Laboratory Animal Center of Xiamen University. During the experiments, the animals were anesthetized with $2 \%-4 \%$ isoflurane and placed on an electric temperature-controlled heating plate. The primary liver xenograft was established by injecting $2 \times 10^{6} \mathrm{HepG} 2$ cells directly into the hepatic lobe. The metastasis models were established by injecting $2 \times 10^{6}$ B16 cells into either the left hepatic lobe (in $10 \mu \mathrm{L}$ of PBS) or the subcapsular spleen (in $50 \mu \mathrm{L}$ of PBS). The hepatic lobe and spleen were exteriorized through a 5-mm incision below the xiphoid or in the left abdomen above the spleen, respectively. The cells were injected into the hepatic or splenic parenchyma using a $1-\mathrm{mL}$ syringe fitted with a 29 -gauge needle. The incision was closed using absorbable sutures.

The mice were imaged $4 \mathrm{~d}$ after tumor cell injection. A mixture of $5 \%$ isoflurane in air was used to anesthetize the animals. The anesthetic state was maintained with $2 \%$ isoflurane and monitored by the respiration rate (25-30 breaths/min). The mice were euthanized when the tumor was larger than $1 \mathrm{~cm}$ in diameter or when the animals displayed tumor-related symptoms such as signs of pain, bleeding, ulcers, necrosis, or weight loss.

\section{In Vitro Experiments and PAI of B16 Cells}

The tissue-mimicking agarose phantom was made using a cylindric glass bottle $\left(3 \mathrm{~cm}^{2}\right.$ in cross section $\times 10 \mathrm{~cm}$ in length). The phantom consisted of $1.5 \%$ agarose per volume, and $20 \%$ intralipid was added during the cooling process before solidification to mimic tissuescattering and -absorption properties. Before in vivo imaging, B16 cell suspensions of varying numbers $\left(0,2.19 \times 10^{2}, 3.5 \times 10^{2}, 4.38 \times\right.$ $10^{2}, 8.75 \times 10^{2}, 1.75 \times 10^{3}, 3.5 \times 10^{3}, 1.75 \times$ $10^{4}$, and $3.5 \times 10^{4}$ ) in $10 \mu \mathrm{L}$ of PBS were imaged to determine the imaging sensitivity. The cells were embedded in a tissue-mimicking phantom ( $n=3$ for each) and imaged photoacoustically at $780 \mathrm{~nm}$.

\section{MRI of Mice In Vivo}

MRI was performed on a BioSpin 9.4-T smallanimal MRI scanner (Bruker) equipped with a horizontal bore (diameter, $30 \mathrm{~cm}$; gradient 
strength, $734 \mathrm{mT} / \mathrm{m}$ ) and fitted with a mouse body coil. T2-weighted axial images were obtained using a spin-echo multiple-slice sequence with the following parameters: repetition time, 2,500 ms; echo time, $33 \mathrm{~ms}$; matrix, $256 \times 256$; field of view, $3 \times 3 \mathrm{~cm}$; signal averaging, 4 ; and section thickness, $1 \mathrm{~mm}$.

\section{Bioluminescence Imaging of Mice In Vivo}

Bioluminescence images were obtained using an IVIS in vivo imaging system (Lumina; Perkin Elmer), which consists of a chargecoupled-device camera to capture both visible-light and luminescent images and an adjustable imaging platform assembled in a lighttight box. The mice were intraperitoneally injected with D-luciferin (150 $\mathrm{mg} / \mathrm{kg}$ ) (Thermo Fisher Scientific) $10 \mathrm{~min}$ before imaging and placed on the stage of the imaging chamber. After acquisition of the photographic images of each mouse, the luminescent images were acquired with an autoexposure setting. The actual exposure time for a whole mouse was $10-30 \mathrm{~s}$.

\section{${ }^{18}$ F-FDG PET/CT Imaging of Mice In Vivo}

An Inveon small-animal PET/CT system (Siemens) was used for whole-body imaging. Each mouse was injected with 3.7-7.4 MBq of ${ }^{18}$ F-FDG (PET Center of the First Affiliated Hospital of Xiamen University) of more than $98 \%$ radiochemical purity in a total volume of 50-80 $\mu \mathrm{L}$ through the caudal vein. A static PET/CT scan of the liver was performed on anesthetized mice $40 \mathrm{~min}$ after ${ }^{18} \mathrm{~F}$-FDG injection. The CT images were acquired by an x-ray camera to determine the orientation of the mice. The PET images were reconstructed using 3-dimensional (3D) ordered-subset expectation maximization combined with attenuation and scattering correction. A region of interest covering the entire tumor was rendered manually on the CT image and was then applied to the corresponding PET images. $\mathrm{SUV}_{\max }$ was calculated for the tumors and normal tissues from the PET images. The ratio of the $\mathrm{SUV}_{\text {max }}$ of the tumor to that of the normal liver tissue was quantified.

\section{Human Sample Collection and Analysis}

Forty specimens of normal and tumor tissues from the liver, stomach, colorectum, and esophagus were collected from 30 patients who underwent tumor resection at the affiliated Zhongshan Hospital of Xiamen University. Their cancer diagnosis had been confirmed by pathologic examination. The samples were collected with the approval of the ethical committee of the Affiliated Zhongshan Hospital of Xiamen University after written informed consent had been obtained from each patient.

The tissue samples were flash-frozen in liquid nitrogen immediately after excision and divided into 3 portions for absorption spectroscopy, PAI, and histology. Light absorption was measured from 300 to $900 \mathrm{~nm}$ at 2-nm increments on triturated identical sample masses using a Multiskan Spectrum (Thermo Fisher). PAI was performed at different wavelengths $(700,750,800,850$, and $900 \mathrm{~nm})$. Depth imaging was standardized in chicken breast of different thicknesses $(0.4,0.8,1.2$, and $1.6 \mathrm{~cm}$ ) at $750 \mathrm{~nm}$. The grayscale PAI scans of the tumors were contoured using MATLAB (version 2018b; The MathWorks Inc.) to determine the boundaries. Dense contours indicated strong tumor infiltration. The intensity and distribution of signal in the images were determined by counting the pixels and plotting them on histograms. Different parameters, including kurtosis, skewness, mean, maximum, and minimum, were calculated.

\section{Immunohistochemistry}

The hypoxic area was visualized by immunohistochemistry using the Hypoxyprobe-1 Plus Kit (Hypoxyprobe Inc.), which detects pimonidazole, a nitroimidazole that selectively accumulates in hypoxic cells and forms stable adducts with protein thiol groups. The tumorbearing mice were intraperitoneally injected with pimonidazole hydrochloride $(60 \mathrm{mg} / \mathrm{kg})$ and sacrificed $1 \mathrm{~h}$ later. The tumor tissues were resected and processed as described above. The sections were incubated sequentially with fluorescein isothiocyanate-conjugated mouse antipimonidazole monoclonal antibody (dilution, 1:200) and with peroxidaseconjugated rabbit anti-fluorescein isothiocyanate secondary antibody (dilution, 1:200) according to kit instructions. To detect tumor angiogenesis, the tissue sections were probed with rabbit anti-CD31 mouse monoclonal antibody (dilution, 1:200; Abcam) and goat antirabbit secondary antibody (dilution, 1:200; KPL). The sections were counterstained with hematoxylin and observed under $\times 20$ magnification. The CD31 or pimonidazole cells were counted in 5 random fields. The percentages of the respective stained positive cells were calculated using the ImageJ immunohistochemistry profiler.

\section{PAI of Hepatic Melanoma Mouse In Vivo}

A pulse laser integrated with a high-frequency ultrasound system (Vevo LAZR-X; FujiFilm VisualSonics), having the advantages of fast imaging and portability, was used to simultaneously acquire PAI scans and ultrasound images (28). A tunable laser (680-970 nm) with a repetition rate of $20 \mathrm{~Hz}$ was integrated into a 256-element linear array with a main frequency of $40 \mathrm{MHz}$. Ultrasound and photoacoustic signals were processed and reconstructed at a workstation. The energy supplied by each pulse of the tunable laser was $1.2 \mathrm{~mJ} / \mathrm{cm}^{2}$, well below the standard set by the American National Standard Institute across the wavelength range. Before imaging, the mice were evenly smeared with medical ultrasound couplant on the target areas and placed on the heating stage connected to an anesthetic catheter. The 3D scanning was controlled by an electric motor with a self-driven step size of $0.14 \mathrm{~mm}$. Oxygen saturation $\left(\mathrm{sO}_{2}\right)$ images were acquired at 750 and 850 $\mathrm{nm}$ and then analyzed by a Vevo LAB tool (FujiFilm VisualSonics). The 3D area ( $>30$ slices) was analyzed by tracking the region of interest. A linear least-squares spectral decomposition algorithm was used to calculate the absorption of endogenous melanin in the liver relative to the total photoacoustic signal (29). Contrasting images of melanin and hemoglobin were obtained on account of their distinct absorption spectra.

A hemispheric PAI system (Nexus 128; Endra) was used to acquire high-resolution images (30). The system consists of a tunable laser beam $(680-950 \mathrm{~nm})$ with a frequency of $20 \mathrm{~Hz}$ and 128 ultrasonic transducers of 5-MHz frequency that are evenly arranged on the surface of a hemispheric bowl. The laser beam irradiates from the bottom of the bowl and is amplified by a concave lens. A custom-made tray with a round slot in the center was placed on top of the bowl to position the mice, and this configuration was confirmed to have a negligible effect on the photoacoustic signal. The bowl and the slot were filled with distilled water to provide acoustic coupling with the imaging target. During imaging, the mice were oriented for optimal alignment. The PAI scans were reconstructed and displayed using OsiriX imaging software (OsiriX Foundation) with a maximum-intensity-projection algorithm. The tumor-to-background ratio was then calculated.

\section{Hematoxylin and Eosin Staining and Immunohistochemistry}

The resected tissues were fixed in $4 \%$ paraformaldehyde, embedded in paraffin, and cut into 5- $\mu \mathrm{m}$ sections. For histologic examination, the sections were stained with hematoxylin and eosin and observed under $\times 5$ magnification (Olympus BX51). Tumor angiogenesis and hypoxic areas were stained with anti-CD31 and antipimonidazole antibody, respectively, by immunohistochemistry.

\section{In Vivo PAI-Guided Surgery}

After the melanoma has grown to approximately $4 \mathrm{~mm}^{3}$, we conducted in vivo surgeries guided by PAI. During the surgery the nude mouse was placed on a mouse table for position adjustment and then anesthetized with $2 \%-4 \%$ isoflurane. For performing melanoma resection surgery monitoring, we used operating scissors, forceps, suture needle, and a surgery needle. The surgery was performed in 5 steps: PAI-guided skin incision, melanoma detection, and imaging (step 1); advancement of the needle toward the tumor (step 2); PAI-guided partial resection of the melanoma 


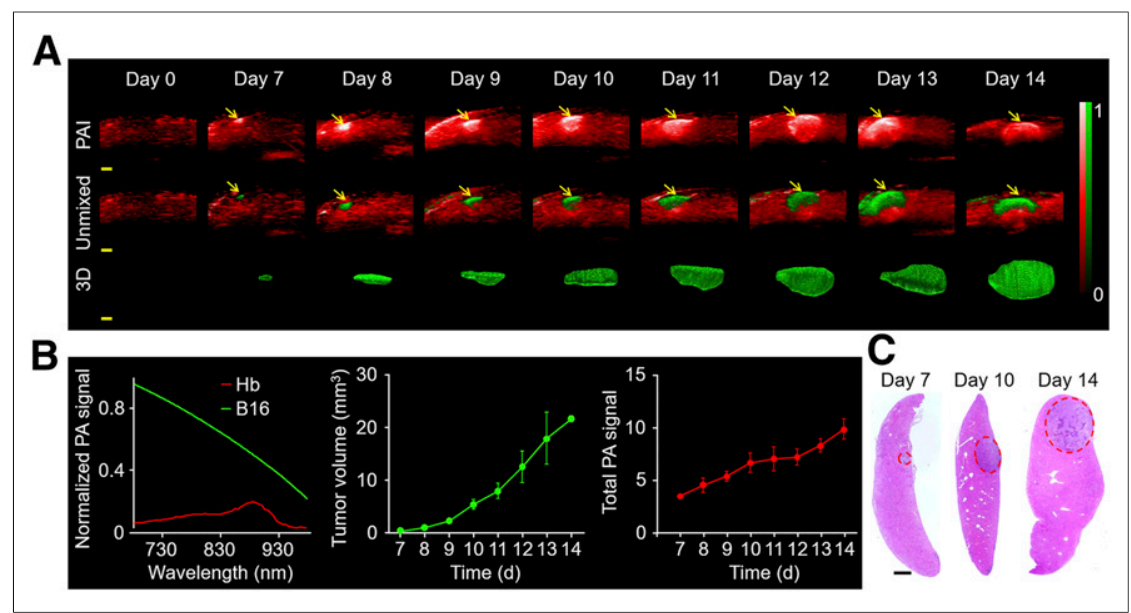

FIGURE 2. PAI displays changes in hepatic melanoma in situ. (A) In vivo PAl of liver before and after hepatic melanoma modeling by hepatic injection at $780 \mathrm{~nm}(n=3)$. Melanoma (green) and hemoglobin (red) were unmixed by multiwavelength PAl at 680,730, 924, and $950 \mathrm{~nm}$. 3D view of tumors is shown. Scale bars in all images are $1 \mathrm{~mm}$. Arrows indicate photoacoustic signal. (B) Normalized photoacoustic signal spectra of hemoglobin and B16. Measurement of tumor volume and photoacoustic signal by PAI. (C) Hematoxylin and eosin staining of tumor at different time points. Scale bars in histologic images are $1 \mathrm{~mm}$. $\mathrm{Hb}=$ hemoglobin; PA = photoacoustic.

time-dependent increase in volume indicated irregular and invasive growth of the tumor, which agreed well with the increasing intratumoral photoacoustic signal (Fig. 2B). Moreover, the detecting limit of PAI was approximately 219 cells in phantoms (Supplemental Fig. 2) (4), and an imaging depth of approximately $7 \mathrm{~mm}$ was displayed in vivo (Supplemental Fig. 3). Taken together, PAI is a noninvasive, highly sensitive, and high-contrast imaging tool that can detect submillimeter-scale tumors at early stages and is a feasible option for direct longterm surveillance.

\section{Comparison of Different Imaging Techniques for Hepatic Metastasis of Melanoma}

To determine the potential clinical value of PAI, we compared it with ultrasound, MRI, bioluminescence imaging, and ${ }^{18} \mathrm{~F}$ FDG PET/CT on the melanoma hepatic-metastasis model. PAI detected the metastatic tumors by the sixth day after modeling and

(step 3); and PAI detection of residual tumor and completion of resection (step 4), followed by skin suturing (step 5).

\section{Statistical Analysis}

The histogram parameters, including kurtosis, skewness, mean, maximum, and minimum, were compared between the poorly and moderately differentiated tumors using the Student $t$ test. Receiveroperating-characteristic curves were plotted for these parameters to assess the area under the curve. The distinguishing parameters with higher areas under the curve (range, $0.5-1$ ) were identified. $P$ values of less than 0.05 were considered statistically significant.

All data analysis was performed using the SPSS software package (version 21.0, IBM Corp.). Data are presented as mean \pm SEM. Twotailed paired $t$ testing was used to compare changes in photoacoustic and PET signals in metastasized tumors.

\section{RESULTS}

\section{PAI of Hepatic Melanoma In Situ}

Mice bearing melanoma in the liver were longitudinally monitored at $780 \mathrm{~nm}$ before and 4-14 d after model establishment to evaluate the feasibility of portable PAI in hepatic melanoma. As shown in Figure 2A, the normal liver displayed a low, homogeneous background signal. On the seventh day after melanoma cell injection, a 4.5 times brighter photoacoustic signal appeared, likely generated by the incipient tumor. The tumor-to-background ratio increased to 6.7 and 9.9 on days 10 and 14, respectively, corresponding to the growing tumor mass. PAI detected hepatic tumor masses as small as approximately $400 \mu \mathrm{m}$ in diameter (Fig. 2A). By taking advantage of the distinct absorption spectra of melanin and hemoglobin (Fig. 2B), the visualization of hepatic melanoma growth against a background of liver was realized by multiwavelength PAI at 680, 730, 924, and $950 \mathrm{~nm}$ (Fig. 2A). The photoacoustic signals displayed were consistent with the unmixed pseudo green signals in Figure 2A, which were further validated as a small tumor by hematoxylin and eosin staining (Fig. 2C). 3D views displayed the morphologic changes of the whole tumor (Fig. 2A). Supplemental Videos 1, 2, and 3 show the reconstructed 3D views of the invasive metastatic tumor in the liver on days 7,10, and 14, respectively. The was synchronously validated by bioluminescence imaging (Fig. 3). Three orthogonal views and 3D PAI scans of the metastatic tumors are shown in Supplemental Figure 4. Ultrasonic imaging of the liver showed even echo signals whereas the metastatic tumors displayed low, heterogeneous signals on the ninth day (Fig. 3). T2-weighted MR images of the same axial plane also showed the tumors to have a high signal intensity against a dark, homogeneous liver background on the ninth day (Fig. 3). Thus, PAI detected hepatic metastases significantly earlier than conventional imaging. PAI also monitored the process of tumorigenesis from engraftment to oncogenesis (Supplemental Fig. 5) and monitored tumorigenesis on day 6, suggesting that PAI may be a visualization tool for early tumorigenesis.

Furthermore, as shown in Figure 4A, both PAI and ${ }^{18}$ F-FDG $\mathrm{PET} / \mathrm{CT}$ detected tumorigenesis in vivo. PAI distinguished the

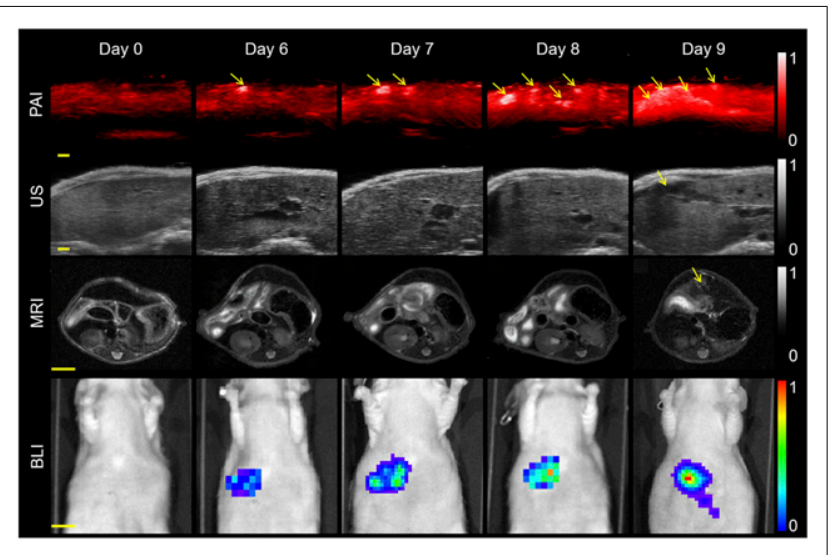

FIGURE 3. Comparison of various imaging modalities. PAI, ultrasound, bioluminescence imaging, and MRI of mouse on different days after tumor cell injection into spleen. Hepatic metastases could be found on PAl, ultrasound, and MRI on days 6, 9, and 9, respectively. Excitation wavelength was $780 \mathrm{~nm}$. Scale bars in PAl scans and ultrasound images are $1 \mathrm{~mm}$. Arrows denote metastatic tumors. Scale bars in MR and bioluminescence images are 5 and $10 \mathrm{~mm}$, respectively. PA = photoacoustic; US = ultrasound. 


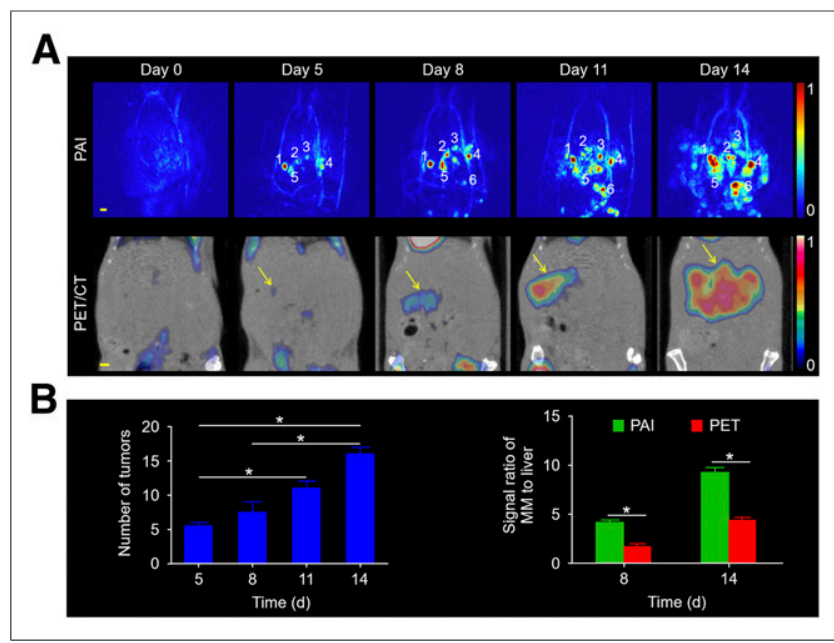

FIGURE 4. PAI and PET for mice with melanoma metastatic to the liver. (A) Images on different days after tumor cell injection at 3-d intervals. Scale bars in all images are $1 \mathrm{~mm}$. (B) Number of tumors over time. Signal ratio for melanoma metastases to liver was quantified in PAI and PET at 8 and $14 \mathrm{~d}$ after tumor cell injection. $\mathrm{MM}=$ melanoma metastasis.

exact size and location of every tumor. However, ${ }^{18}$ F-FDG PET indicated only the presence of tumors. The number of tumors (Fig. 4B) increased from days 5 to 14 . The tumor-to-background ratios for PAI were approximately 2.3 and 2.1 times higher than those for ${ }^{18}$ F-FDG PET on days 8 and 14, respectively (Fig. 4B). Supplemental Videos 4 and 5 show multiple views of the metastatic tumors and the changes in their morphology in 3 dimensions at 1 and 2 wk. These results indicate high sensitivity and resolution for PAI in screening for melanoma micrometastases in the liver.

\section{Monitoring of Oxygenation Dynamics in Metastatic Hepatic Carcinoma with PAI}

Functional imaging of PAI not only could detect pharmacokinetics through indocyanine green (Supplemental Fig. 6) but also could evaluate $\mathrm{sO}_{2}$ metabolism without labeling. As shown in Figure $5 \mathrm{~A}$, the tumors showed a gradual increase in $\mathrm{sO}_{2}$, with clear boundaries against a homogeneous background of the liver. However, the intratumoral change in $\mathrm{sO}_{2}$ was inconsistent because of heterogeneous tumor growth (31), with lower levels in the center than in the margin. Average $\mathrm{sO}_{2}$, which was integrally quantified, increased constantly till day 10 . Positive immunostaining of CD31 in the tumor tissue sections confirmed angiogenesis, and the percentage of tumor angiogenesis showed an increasing trend (Fig. 5B). These findings suggest that the oxygen requirement for the proliferating tumor cells with high metabolic rates was supplied by angiogenesis. The gradient of $\mathrm{sO}_{2}$ showed a downward trend, implying that a small number of tumor cells may have hypoxia. Positive immunostaining of pimonidazole in the tumor tissue sections also confirmed tumor cell hypoxia, and the percentage of hypoxic areas showed an increasing trend (Fig. 5B). Panoramic views of stained tumor sections are shown in Supplemental Figure 7. These results indicate that PAI can be used to detect $\mathrm{sO}_{2}$ and oxygen metabolism in the tumors-valuable parameters to establish the therapeutic regimen and make a clear prognostic assessment (32).

\section{Detection of Clinical and Preclinical Samples by PAI}

Experimental results for samples from 9 patients with primary hepatic carcinoma are shown in Figure 6A. The margins of the tumor and normal tissue were determined by bright photoacoustic signal due to blood chromophores with optical absorption properties. In addition, tumor tissues from stomach, colorectum, and esophagus were imaged by PAI (Supplemental Figs. 8 and 9), which showed better depth imaging (Supplemental Fig. 10). The grayscale PAI scans were analyzed to determine the tumor boundaries (Supplemental Fig. 11) and signal distribution (Supplemental Figs. 12 and 13). The results for preclinical samples from mouse melanoma metastatic to the liver are displayed in Figure 6B. Photographs of metastatic melanoma from frozen sections were consistent with melanoma signal from PAI. Therefore, our results suggest that PAI has potential for detecting tumor and guiding tumor surgery.

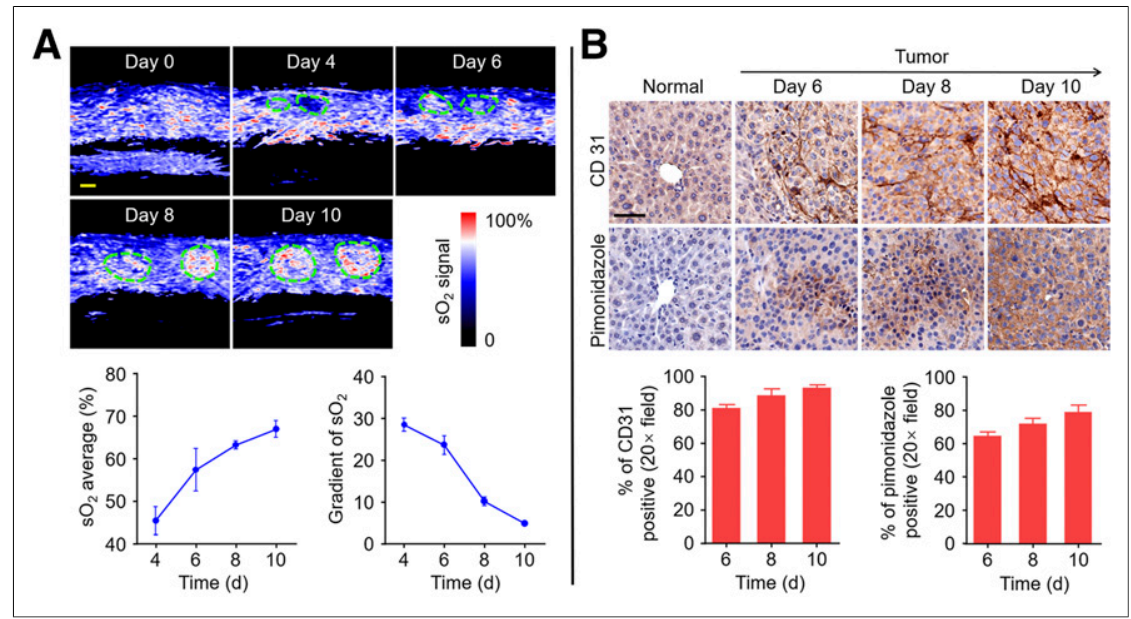

FIGURE 5. Monitoring of functional parameters in tumor and normal tissue. (A) Increase in tumor blood oxygenation from days 4 to 10. Region of interest represents tumor. Scale bars in $\mathrm{sO}_{2}$ images are $1 \mathrm{~mm}$. $\mathrm{sO}_{2}$ average and gradient of $\mathrm{sO}_{2}$ are quantified. (B) Representative immunohistochemistry images of slices collected from normal-mouse liver and melanoma-mouse liver on days 6, 8, and 10 after injection of B16 cells. Nuclei, tumor angiogenesis, and tumor hypoxic areas are stained with hematoxylin, antiCD31 antibody, and antipimonidazole antibody, respectively. Scale bars in immunohistochemistry images are $50 \mu \mathrm{m}$. Tumor angiogenesis and hypoxic areas from immunohistochemistry images are quantified.

\section{PAI-Guided Surgery of Hepatic Metastases}

We further examined the capability of PAI for intraoperative guidance, as shown by the 2-dimensional images in Figure 7. Preoperative PAI was first performed to determine the tumor location (step 0). The figure shows a typical reconstructed $3 \mathrm{D}$ image of the metastatic tumor, which provided the surgeon with a profile of the tumor in the surgical region. The surgical margins were then indicated using a steel needle (step 1). The needle and tumor were clearly visible as the needle continually advanced to the tumor boundary in steps $2-5$ (Supplemental Video 6). We initially performed a partial resection with PAI guidance; the residual tumor is shown in step 6 in the figure. The tumor was completely resected subsequently, with no residue detected in step 7. Ex vivo liver lobes were stacked by inserting a needle into the upper lobe and were placed in the 


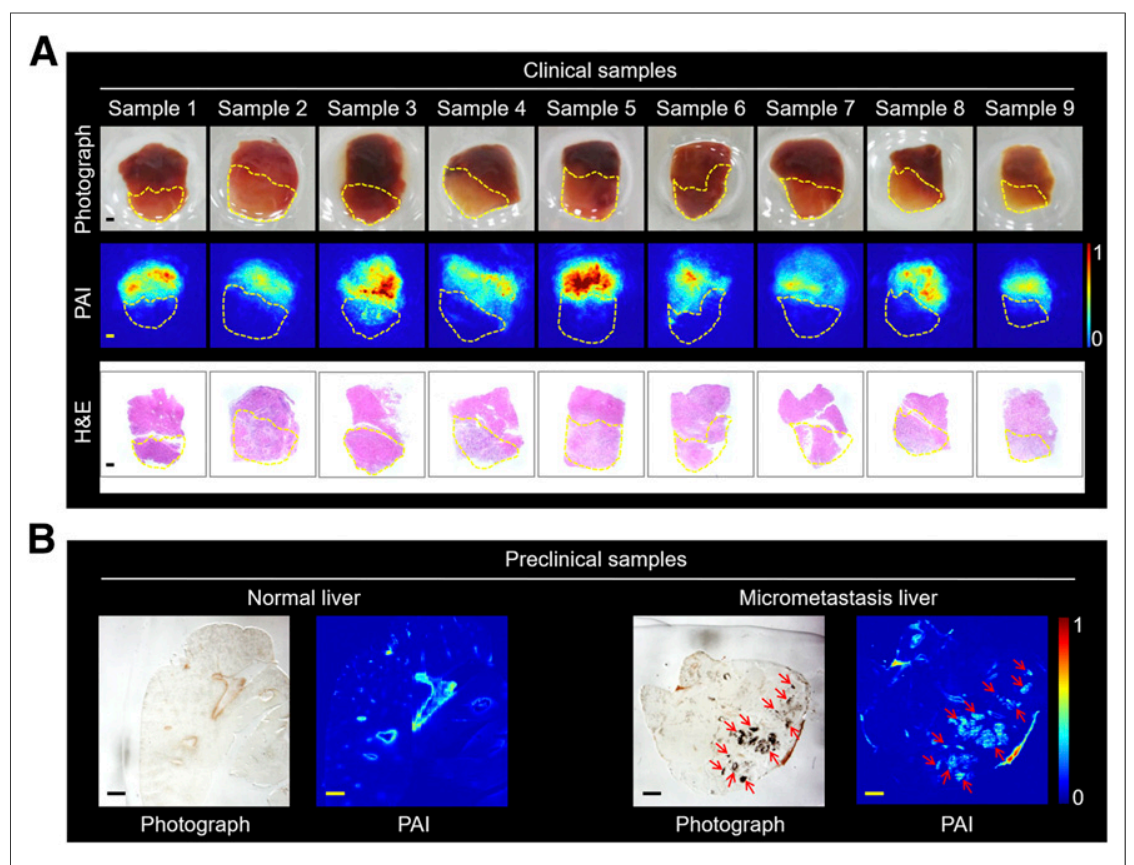

FIGURE 6. PAI of clinical and preclinical samples. (A) Photographs of tumor samples (in ambient normal tissue) from 9 patients, along with PAl scans and hematoxylin and eosin staining. Region of interest represents tumor. Scale bars are $1 \mathrm{~mm}$. (B) Photographs and PAI of normal and melanoma micrometastasis liver slices. Arrows denote tumor. Thickness of liver slice is $50 \mu \mathrm{m}$. Scale bars are $1 \mathrm{~mm} . \mathrm{MM}=$ melanoma metastasis.

tray and imaged at $780 \mathrm{~nm}$. The maximum imaging depth reached $11 \mathrm{~mm}$, with satisfactory contrast (Supplemental Fig. 14). With these results all taken together, intraoperative PAI can be said to effectively locate tumors, identify residual masses, and guide surgical resection.

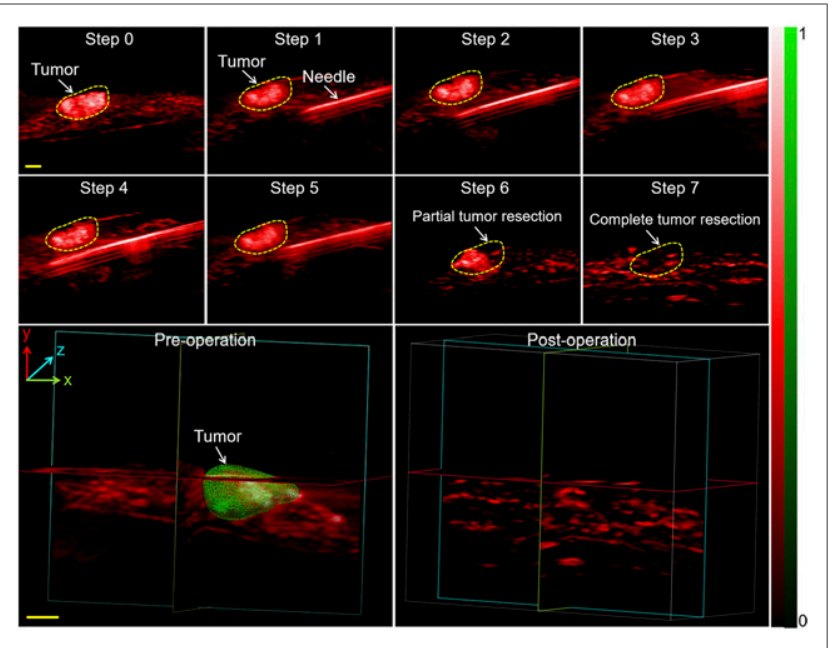

FIGURE 7. Needle insertion and PAl-guided tumor resection in vivo. Shown are PAl scans of metastatic tumor after laparotomy (step 1), advancement of needle toward tumor (step 2), partial resection (step 3), and complete resection (step 4) with PAl guidance. 3D PAl scans before and after resection are displayed. 3D melanoma (green) and hemoglobin (red) distributions were estimated by spectral unmixing analyses from spectroscopic acquisitions at 680,730, 924, and 950 $\mathrm{nm}$. Scale bars in all images are $1 \mathrm{~mm}$. H\&E = hematoxylin and eosin staining.

\section{DISCUSSION}

We used novel PAI technology to detect micrometastatic melanoma in the liver of live mice. The PAI system integrates high temporal and spatial resolution, deep penetration, multiple contrasts, and high sensitivity, therefore capturing structural and functional images of small animals. As a completely noninvasive, fast imaging technique, it is also suitable for long-term monitoring. Our results demonstrate that PAI has the ability to monitor tumor volume changes and metastatic nodes, thus opening a new window for medical researchers to treat and diagnose patients without the danger of the ionizing radiation from CT or PET/CT (33). Alternatively, PAI might be used as a visualization tool for early stages of tumor formation in the liver. Moreover, by clearly distinguishing the blood vessels and liver of mice, PAI might be used to directly diagnose pathologic changes in the liver.

We illustrated how PAI can show the formation process of microtumors, and we established a tool for quantitative evaluation of micrometastasis. PAI could detect microscopic melanoma as small as approximately $400 \mu \mathrm{m}$ in vivo and as few as approximately 219 melanoma cells in vitro. We detected microtumors in vivo using PAI technology by showing a stronger photoacoustic signal than that before inoculation. In contrast, neither ultrasound nor MRI was able to detect any changes in the liver at early stages. Therefore, PAI is an effective tool for the quantitative evaluation of micrometastasis and can be used as a complement to ultrasound, MRI, and PET.

Tumor development is accompanied by microvessel regeneration and local hypoxia. Assessing the hemodynamic response to oxygen is an effective means to monitor tumor metabolism (34). Using different wavelengths, PAI achieved functional images of tumors in mouse liver. The increase in $\mathrm{sO}_{2}$ during tumor growth can be presumed to be due to tumor microvessels in the early stages (35). The decrease in the gradient of $\mathrm{sO}_{2}$ suggests hypoxia in the tumor $(21,36)$. In addition, PAI showed the pharmacokinetics in both mouse liver and internal organs with the assistance of indocyanine green (37). Further clinical application of PAI is conceivable and is being actively pursued, such as to design optimal dosing regimens and administration.

Previous studies have shown that PAI can identify tumors beneath the surface in normal brain tissue and even guide resection of larger tumors (26). Our portable PAI was able to visualize tumor localization and excision in real time during the operation, as well as any residual cancer tissue within the incision margin after resection. Therefore, portable PAI is a highly promising technique that can provide visual guidance during surgery. In addition, intraoperative PAI can potentially detect deeper tumors and improve not only their detection but their complete resection.

The feasibility of the PAI system in the surgical environment was successfully evaluated by in vivo surgery, including image-guided melanoma excision. However, 3 improvements are necessary for future clinical applications. The first of these is a change in the material of the needle to enable monitoring of the movement of surgical instruments 
in deeper layers of tissues. For example, ceramic scalpels may be used for PAI during surgery $(38,39)$. The second improvement is an increase in the laser power to achieve a greater imaging depth. The third is miniaturization of the photoacoustic probe, while retaining adequate signal sensitivity, to allow sufficient space to perform the surgery.

\section{CONCLUSION}

PAI as a new imaging method with high sensitivity and specificity can monitor microtumors $(<1 \mathrm{~mm})$ that cannot be detected by traditional imaging techniques, realizing the early diagnosis of tumors and helping doctors plan treatment as early as possible. Under precise detection conditions, PAI can be further developed to help guide tumor resection by reducing residues. Although this study focused on melanoma metastatic to the liver, we believe that this method can be extended to other tumors, such as glioma and breast cancer.

\section{DISCLOSURE}

This work was supported by the National Science Foundation of China (81922034 and 91859113), the Fundamental Research Funds for the Central Universities (20720170065), the Science Fund for Distinguished Young Scholars of Fujian Province (2018J06024), and the Science Foundation of Fujian Province (2014Y2004). No other potential conflict of interest relevant to this article was reported.

\section{KEY POINTS}

QUESTION: Can PAI be used as a label-free, noninvasive, affordable, and clinically friendly nonionization biomedical imaging modality for early detection of deep hepatic micrometastases and for guiding tumor resection?

PERTINENT FINDINGS: PAl achieved label-free and noninvasive detection of small $(\sim 00 \mu \mathrm{m})$ and deep $(\sim 7 \mathrm{~mm})$ tumors in vivo and surgical resection of submillimeter tumors based on structural and functional imaging features, as verified by histopathologic methods.

IMPLICATIONS FOR PATIENT CARE: PAI, a robust surveillance technique with high resolution and sensitivity, can guide surgical removal of submillimeter tumors to improve patient outcomes in the future.

\section{REFERENCES}

1. Siegel RL, Miller KD, Jemal A. Cancer statistics, 2017. CA Cancer J Clin. 2017;67:7-30.

2. Chambers AF, Groom AC, MacDonald IC. Dissemination and growth of cancer cells in metastatic sites. Nat Rev Cancer. 2002;2:563-572.

3. Welch DR, Hurst DR. Defining the hallmarks of metastasis. Cancer Res. 2019; 79:3011-3027.

4. Neuschmelting V, Lockau H, Ntziachristos V, Grimm J, Kircher MF. Lymph node micrometastases and in-transit metastases from melanoma: in vivo detection with multispectral optoacoustic imaging in a mouse model. Radiology. 2016;280:137-150.

5. Agarwala SS, Eggermont AM, O'Day S, Zager JS. Metastatic melanoma to the liver: a contemporary and comprehensive review of surgical, systemic, and regional therapeutic options. Cancer. 2014;120:781-789.

6. Carreno BM, Garbow JR, Kolar GR, et al. Immunodeficient mouse strains display marked variability in growth of human melanoma lung metastases. Clin Cancer Res. 2009;15:3277-3286.

7. Tawbi HA, Forsyth PA, Algazi A, et al. Combined nivolumab and ipilimumab in melanoma metastatic to the brain. N Engl J Med. 2018;379:722-730.

8. Einhorn LH, Burgess MA, Gottlieb JA. Metastatic patterns of choroidal melanoma. Cancer. 1974;34:1001-1004.

9. Pawlik TM, Zorzi D, Abdalla EK, et al. Hepatic resection for metastatic melanoma: distinct patterns of recurrence and prognosis for ocular versus cutaneous disease. Ann Surg Oncol. 2006;13:712-720.

10. Balch CM, Soong SJ, Gershenwald JE, et al. Prognostic factors analysis of 17,600 melanoma patients: validation of the American Joint Committee on Cancer melanoma staging system. J Clin Oncol. 2001;19:3622-3634.
11. Mehlen P, Puisieux A. Metastasis: a question of life or death. Nat Rev Cancer. 2006;6:449-458

12. Sahani DV, Bajwa MA, Andrabi Y, Bajpai S, Cusack JC. Current status of imaging and emerging techniques to evaluate liver metastases from colorectal carcinoma. Ann Surg. 2014;259:861-872.

13. Wang LV, Wu H-i. Biomedical Optics: Principles and Imaging. Hoboken, NJ: Wiley; 2007:283-284.

14. Wang LV, Hu S. Photoacoustic tomography: in vivo imaging from organelles to organs. Science. 2012;335:1458-1462.

15. Liu Y, Nie L, Chen X. Photoacoustic molecular imaging: from multiscale biomedical applications towards early-stage theranostics. Trends Biotechnol. 2016;34:420-433.

16. Staley J, Grogan P, Samadi AK, Cui H, Cohen MS, Yang X. Growth of melanoma brain tumors monitored by photoacoustic microscopy. J Biomed Opt. 2010; 15:040510.

17. Wang B, Su JL, Amirian J, Litovsky SH, Smalling R, Emelianov S. Detection of lipid in atherosclerotic vessels using ultrasound-guided spectroscopic intravascular photoacoustic imaging. Opt Express. 2010;18:4889-4897.

18. Viator JA, Komadina J, Svaasand LO, Aguilar G, Choi B, Stuart Nelson J. A comparative study of photoacoustic and reflectance methods for determination of epidermal melanin content. J Invest Dermatol. 2004;122:1432-1439.

19. Stoffels I, Morscher S, Helfrich I, et al. Metastatic status of sentinel lymph nodes in melanoma determined noninvasively with multispectral optoacoustic imaging. Sci Transl Med. 2015;7:319ra8.

20. Li L, Zhu L, Ma C, et al. Single-impulse panoramic photoacoustic computed tomography of small-animal whole-body dynamics at high spatiotemporal resolution. Nat Biomed Eng. 2017;1:0071.

21. Yao J, Maslov KI, Zhang Y, Xia Y, Wang LV. Label-free oxygen-metabolic photoacoustic microscopy in vivo. J Biomed Opt. 2011;16:076003.

22. Frangioni JV. In vivo near-infrared fluorescence imaging. Curr Opin Chem Biol. 2003;7:626-634

23. Kularatne SA, Thomas M, Myers $\mathrm{CH}$, et al. Evaluation of novel prostate-specific membrane antigen-targeted near-infrared imaging agent for fluorescence-guided surgery of prostate cancer. Clin Cancer Res. 2019;25:177-187.

24. Wilson KE, Bachawal SV, Willmann JK. Intraoperative resection guidance with photoacoustic and fluorescence molecular imaging using an anti-B7-H3 antibodyindocyanine green dual contrast agent. Clin Cancer Res. 2018;24:3572-3582.

25. Wong TTW, Zhang R, Hai P, et al. Fast label-free multilayered histology-like imaging of human breast cancer by photoacoustic microscopy. Sci Adv. 2017;3: e1602168.

26. Kircher MF, de la Zerda A, Jokerst JV, et al. A brain tumor molecular imaging strategy using a new triple-modality MRI-photoacoustic-Raman nanoparticle. Nat Med. 2012;18:829-834.

27. Moore C, Jokerst JV. Strategies for image-guided therapy, surgery, and drug delivery using photoacoustic imaging. Theranostics. 2019;9:1550-1571.

28. Wu Z, Duan F, Zhang J, Li S, Ma H, Nie L. In vivo dual-scale photoacoustic surveillance and assessment of burn healing. Biomed Opt Express. 2019;10: 3425-3433.

29. Kim S, Chen YS, Luke GP, Emelianov SY. In vivo three-dimensional spectroscopic photoacoustic imaging for monitoring nanoparticle delivery. Biomed Opt Express. 2011;2:2540-2550.

30. Lv J, Peng Y, Li S, et al. Hemispherical photoacoustic imaging of myocardial infarction: in vivo detection and monitoring. Eur Radiol. 2018;28:2176-2183.

31. Reina-Campos M, Moscat J, Diaz-Meco M. Metabolism shapes the tumor microenvironment. Curr Opin Cell Biol. 2017;48:47-53.

32. Menon C, Fraker DL. Tumor oxygenation status as a prognostic marker. Cancer Lett. 2005;221:225-235.

33. Hong H, Zhang L, Xie F, et al. Rapid one-step ${ }^{18} \mathrm{~F}$-radiolabeling of biomolecules in aqueous media by organophosphine fluoride acceptors. Nat Commun. 2019;10:989.

34. Skala MC, Fontanella A, Lan L, Izatt JA, Dewhirst MW. Longitudinal optical imaging of tumor metabolism and hemodynamics. J Biomed Opt. 2010;15:011112.

35. Nie L, Huang P, Li W, et al. Early-stage imaging of nanocarrier-enhanced chemotherapy response in living subjects by scalable photoacoustic microscopy. ACS Nano. 2014;8:12141-12150.

36. Gerling M, Zhao Y, Nania S, et al. Real-time assessment of tissue hypoxia in vivo with combined photoacoustics and high-frequency ultrasound. Theranostics. 2014;4:604-613.

37. Taruttis A, Morscher S, Burton NC, Razansky D, Ntziachristos V. Fast multispectral optoacoustic tomography (MSOT) for dynamic imaging of pharmacokinetics and biodistribution in multiple organs. PLoS One. 2012;7:e30491.

38. Klocke F, Linke B, Schluetter D. Development of a reliable grinding procedure for ceramic medical instruments. Prod Eng Res Devel. 2010;4:571-579.

39. Burbelo RM, Gulyaev AL, Robur LI, Zhabitenko MK, Atamanenko BA, Kryl YA. Photoacoustic visualization of residual stress in ceramic material. Journal de Physique IV Colloque. 1994;04:C7-311-C7-314. 